CRETE 2010

2nd International Conference on

Hazardous and Industrial Waste Management

5-8 October 2010

Chania, Crete,

Greece

Organized by:

Technical University of Crete (GR)

University of Padua (IT)

Hamburg University of Technology (DE)

IWWG - International Waste Working Group 


\title{
CHARACTERIZATION OF CHARS PRODUCED IN THE CO-PYROLYSIS OF DIFFERENT WASTES: DECONTAMINATION STUDY
}

\author{
M. BERNARDO ${ }^{a}$, N. LAPA ${ }^{a}$, M. GONÇALVES ${ }^{a}$, R. BARBOSA ${ }^{a}$, B. MENDES ${ }^{a}$, \\ F. PINTO ${ }^{\mathrm{b}}$
}

${ }^{a}$ Ubia, Faculdade de Ciências e Tecnologia, Universidade Nova de Lisboa, 2829516 Caparica, Portugal

${ }^{b}$ LNEG-DEECA, Ed. J, Estrada do Paço do Lumiar 22, 1649-038 Lisboa, Portugal

Summary: The present work is devoted to the study of chars obtained in the co-pyrolysis of plastics, biomass and tyres wastes. The chars were extracted with several organic solvents in order to assess the more efficient in reducing the organic load of the chars and, therefore, their toxicity. The ability of each selected extractant to remove toxic pollutants was evaluated by comparing the extracts yield and thoroughly characterizing the different crude extracts obtained by combining chemical analysis and toxicity bioassays. Also, the mineral composition of the treated and non treated chars was assessed. The results will allow to conclude which solvent should be used in the decontamination of the pyrolysis chars.

\section{INTRODUCTION}

Plastics, biomass and tyre wastes are three types of residues that presently pose difficulties when addressing their final destination. The world's annual production of plastic materials has reached around 245 million tonnes today with Europe representing 25\% of the global plastics production (APME, 2009) and, at present, over 1 billion tyres are sold worldwide each year and subsequently just as many fall into the category of end of life tyres (ETRMA, 2010). Also, biomass wastes are produced in huge amounts from different sources and recovery routes have to be developed for these wastes in order to reclaim its organic content in an energetic useful way (Zhang et al., 2010).

The Directive 2008/98/EC (EC, 2008) of the European Parliament clearly states that, by 2020, all solid waste streams should be diverted towards thermal and/or mechanical treatment and energy recovery.

Thermal treatment of wastes, like gasification or pyrolysis, is considered to be one of the best treatment methods since the deposition in landfills and/or thermal treatment by incineration raises lot of controversy about the emissions of toxic compounds. Pyrolysis is a process of thermal degradation of wastes in the absence of air to produce recyclable products (char, oil and combustible gases). The liquid oil has properties and composition similar to the petroleum 
derived fuels and can be used as raw material for the petrochemical industry. The gas fraction can also be used to provide energy for the pyrolysis process. Concerning the solid fraction (char), it can be used as carbon black or alternatively, upgraded to activated carbon (Buah et al., 2007). These chars are mainly composed of a carbon-rich matrix that contains a broad range of toxic substances including heavy metals and organic compounds distributed by a porous structure (Helleur et al., 2001). Therefore, they have a toxicity potential that must be assessed in order to define their safe reutilization or disposal.

The present work is devoted to the study of chars obtained in the co-pyrolysis of plastics, biomass and tyres wastes. The composition of these chars is not yet well studied and only recent an attempt was made by Bernardo et al. (2010) to provide some information about the composition and risk assessment of these materials.

The objectives of this work were: to perform solvent extractions, using different solvents, in chars obtained from the co-pyrolysis of plastics, tires and biomass waste; to evaluate the extraction efficiency by characterising the different solvent extracts obtained as well as the extracted chars; to perform a chemical and bioassay fractionation in each of the solvent extracts in order to conclude the more efficient solvent to be used in the decontamination of the pyrolysis chars.

\section{MATERIALS AND METHODS}

\subsection{Pyrolysis chars}

The char samples were obtained in the co-pyrolysis of a waste mixture composed of $30 \%(\mathrm{w} / \mathrm{w})$ pine biomass, $30 \%(\mathrm{w} / \mathrm{w})$ used tyres and $40 \%(\mathrm{w} / \mathrm{w})$ plastics. The mixture of plastics was $56 \%$ $(\mathrm{w} / \mathrm{w})$ polyethylene, $27 \%(\mathrm{w} / \mathrm{w})$ polypropylene and $17 \%(\mathrm{w} / \mathrm{w})$ polystyrene, simulating the composition of the plastic fraction present in Portuguese Municipal Solid Wastes (MSW).

Pyrolysis experiments were conducted in a $1 \mathrm{~L}$ autoclave, during $15 \mathrm{~min}$ at a temperature of $420^{\circ} \mathrm{C}$ with an initial pressure of $0.41 \mathrm{MPa}$. More information about the pyrolysis installation and experiments can be found in previous papers (Bernardo et al., 2010; Paradela et al., 2009).

\subsection{Chars extraction: solvents selection}

The pyrolysis chars were submitted to a soxhlet extraction according to an adaptation of the EPA 3540C Method (USEPA, 1996a). Several organic solvents with different polarities were used: hexane, dichloromethane, a mixture of $1: 1(\mathrm{v} / \mathrm{v})$ of hexane:acetone and ethanol.

Also, a sequential extraction of hexane $\rightarrow$ mixture of $1: 1(\mathrm{v} / \mathrm{v})$ hexane:acetone $\rightarrow$ ethanol was performed in order to achieve a higher extraction yield.

The crude extract solutions obtained were concentrated and dried using a vacuum rotary evaporator. All extracts were placed at room temperature before weighing gravimetrically to determine the extraction yields.

\subsection{Determination of the content in heavy metals in the chars}

The chars were submitted to a previous digestion made with hydrogen peroxide $30 \%(\mathrm{v} / \mathrm{v})$ in a heated bath at a temperature of $95^{\circ} \mathrm{C}$ and then with aqua regia $\left(\mathrm{HCl}: \mathrm{HNO}_{3}, 3: 1, \mathrm{v} / \mathrm{v}\right)$ at the same temperature. Finally, a microwave acidic digestion with aqua regia in closed PTFE vessels was used to complete the solubilization of the inorganic components of the samples. 
A selected group of heavy metals ( $\mathrm{Cd}, \mathrm{Cr}, \mathrm{Ni}, \mathrm{Cu}, \mathrm{Zn}, \mathrm{Pb}, \mathrm{Hg}$ and $\mathrm{As}$ ) were quantified in the digested samples using a Thermo Elemental Solaar atomic absorption spectrometry equipment.

\subsection{Toxicity testing: bioassays}

All the crude extract solutions obtained with the different organic solvents were analysed for ecotoxicity with the standardized Microtox ${ }^{\circledR}$ bioassay based on the luminescence inhibition of the bacterium Vibrio fischeri when exposed to toxicants (Azur Environmental Microtox ${ }^{\circledR}$ system). The Microtox ${ }^{\circledR}$ toxicity test adequate for organic extracts was followed in this work according the manufacturer's protocol (Microtox, 1992). The extract solutions were solventexchanged to the organic solvent dimethylsulfoxide (DMSO). The luminescence inhibition of $V$. fischeri was evaluated for an exposure period of 5,15 , and $30 \mathrm{~min}$. The $\mathrm{EC}_{50}$ values (effective concentration of toxicant resulting in a $50 \%$ decrease in bioluminescence) of the DMSO crude extracts were expressed as mass per liter of diluent.

\subsection{Fractionation of the most toxic crude extract - chemical analysis}

The crude extract that presented the highest toxicity was fractionated into aliphatic, aromatic and polar fractions according to the EPA 3611B Method (USEPA, 1996b). The extract was fractionated on a glass column packed with neutral $\mathrm{pH}$ alumina and the aliphatic, aromatic and polar fractions were eluted with hexane, dichloromethane and methanol, respectively.

The aliphatic and aromatic fractions were analysed for chemical composition using gas chromatography with mass spectrometry. The compounds of interest were identified by comparison of the retention times and mass spectra of the compounds with standards and also by tentatively identification by the comparison of their mass spectra with references from the Wiley and NIST spectra libraries.

The polar fraction was not analysed by GC-MS and will be object of study in a future work.

\section{RESULTS AND DISCUSSION}

\subsection{Extract yield for different extraction solvents}

The effects of organic solvents on the extraction yield were studied and the results are presented in Table 1.

Table 1. Extract yields obtained with the different extraction solvents.

\begin{tabular}{cc}
\hline Extraction solvent & Extract yield (\% g/g sample) \\
\hline Hexane & 58.1 \\
Dichloromethane & 54.9 \\
Hexane:acetone $(1: 1 \mathrm{v} / \mathrm{v})$ & 40.6 \\
Ethanol & 32.6 \\
\hline
\end{tabular}

The most effective extractants for single extractions were hexane and dichloromethane with extract yields of $58.1 \%$ and $54.9 \%$, respectively, which shows that most of the pyrolysis chars organic components are non-polar or with low polarity. The extract yields decreases with the 
solvent polarity, with ethanol being the solvent with the worst removal efficiency (32.6\%). It is shown that solvent polarity plays an important role in decreasing the solubility of organic contaminants from the pyrolysis chars. Nevertheless, even with the increasing polarity of the solvents the extracts yields were considerable high, reflecting the complexity and diversity of char's composition.

In order to achieve a more efficient organic removal, a combination of individual extractants was selected to sequentially extract all contaminants. The combination of organic solvents selected for the sequential extraction and the extract yields obtained in each extraction step is listed in Table 2.

In the first step of extraction, hexane was chosen to extract most of the organic contaminants, since it was the organic solvent that, individually, allowed to achieve the highest extract yield. In the second extraction step, with the mixture 1:1 (v/v) of hexane and acetone, a significant removal of organic compounds was still observed with an extract yield of 17.2\%. Probably, the more polar components were extracted in this step. The last extraction step with ethanol removed a small amount of organic contaminants, with an extract yield not significant $(1.9 \%)$. No advantages could be observed in performing this last extraction step.

The global extract yield of the sequential extraction was around 65.9\%, whereby a combination of extraction solvents, namely, an extraction with hexane followed by an extraction of hexane:acetone mixture, could be used to remove more efficiently different classes of organic contaminants.

Table 2. Sequential extraction experiment and extract yields obtained in each extraction step.

\begin{tabular}{ccc}
\hline \multirow{2}{*}{ Extraction step } & Extraction solvent & Extract yield (\% g/g sample) \\
\hline 1 & Hexane & 58.1 \\
2 & Hexane:Acetone (1:1 v/v) & 17.2 \\
3 & Ethanol & 1.9 \\
\hline
\end{tabular}

\subsection{Mineral composition of the chars}

Table 3 shows the content of heavy metals in chars (extracted and non extracted). The heavy metals cadmium $(\mathrm{Cd})$, chromium $(\mathrm{Cr})$ and nickel $(\mathrm{Ni})$ were not detected in any of the chars. Zinc $(\mathrm{Zn})$ and lead $(\mathrm{Pb})$ were detected in the chars in significant concentrations. Plastic additives in the plastic wastes used in the co-pyrolysis process might be a major source for metals contamination in chars. $\mathrm{Zn}$ is present with major amounts in tyres since zinc oxide is added as an activator during the vulcanizing process (Adachia and Tainosho, 2004), thus the introduction of tyres wastes in the pyrolysis mixture can be the source of chars contamination with this metal.

In general, the concentrations of the heavy metals in the extracted chars are higher than in the raw char. This could be attributed to a concentration effect associated with the extraction treatment with solvents in which occurs mass reduction. Moreover, in the raw char some metals might be strongly associated with the organically char matrix and were not complete solubilised in the acid digestion whereby they might be sub estimated. After the treatment with the organic solvents, the carbon structure of the chars may have suffered some changes and the solubility of the metals may have increased. No significant differences can be observed in the metal content of the different extracted chars, what somehow was expected. Therefore, the small differences in the heavy metals amount might be because of the heterogeneities of the chars matrix.

Zinc represents the major problem taking into account it higher concentration in the treated chars. However, it was previously demonstrated that Zn mobility/leachability (Bernardo et al., 
2010) is suppressed in the extracted pyrolysis chars. Also, previous studies (Hwang et al., 2007; Kistler et al., 1987) have shown that pyrolysis may have a positive effect on the immobilization of heavy metals in solid chars.

Table 3. Inorganic characterization of the raw and extracted chars.

\begin{tabular}{ccccccc}
\hline $\begin{array}{c}\text { Metals } \\
(\mathrm{mg} / \mathrm{kg})\end{array}$ & Raw char & $\begin{array}{c}\text { Char } \\
\text { Hex }\end{array}$ & $\begin{array}{c}\text { Char } \\
\text { DCM }\end{array}$ & $\begin{array}{c}\text { Char } \\
\text { Hex:Acet }\end{array}$ & $\begin{array}{c}\text { Char } \\
\text { Et }\end{array}$ & $\begin{array}{c}\text { Char } \\
\text { Seq }\end{array}$ \\
\hline $\mathrm{Cd}$ & $<0.6$ & $<0.6$ & $<0.6$ & $<0.6$ & $<0.6$ & $<0.6$ \\
$\mathrm{~Pb}$ & $23.6 \pm 6.6$ & $73.3 \pm 2.8$ & $61.5 \pm 0.3$ & $61.6 \pm 2.0$ & $55.1 \pm 5.9$ & $76.6 \pm 5.9$ \\
$\mathrm{Zn}$ & $3615 \pm 539$ & $12142 \pm 221$ & $9868 \pm 352$ & $8388 \pm 1267$ & $6686 \pm 612$ & $9128 \pm 183$ \\
$\mathrm{Cu}$ & $1.9 \pm 1.5$ & $3.8 \pm 0.6$ & $4.0 \pm 2.6$ & $<1.0$ & $3.4 \pm 0.5$ & $4.5 \pm 2.5$ \\
$\mathrm{Cr}$ & $<4.5$ & $<4.5$ & $<4.5$ & $<4.5$ & $<4.5$ & $<4.5$ \\
$\mathrm{Ni}$ & $<1.8$ & $<1.8$ & $<1.8$ & $<1.8$ & $<1.8$ & $<1.8$ \\
$\mathrm{Hg}$ & $0.17 \pm 0.09$ & $0.09 \pm 0.02$ & $0.22 \pm 0.01$ & $0.27 \pm 0.10$ & $0.24 \pm 0.02$ & $0.25 \pm 0.05$ \\
$\mathrm{As}$ & $0.09 \pm 0.04$ & $0.21 \pm 0.07$ & $0.12 \pm 0.03$ & $0.30 \pm 0.08$ & $0.24 \pm 0.05$ & $0.29 \pm 0.01$ \\
\hline
\end{tabular}

Raw char - char non extracted; Char DCM - char extracted with dichloromethane; Char Hex - char extracted with hexane; Char Hex:Acet - char extracted with the mixture 1:1 (v/v) of hexane and acetone; Char Et - char extracted with ethanol; Char Seq - char sequentially extracted).

\subsection{Toxicity of crude solvent extracts}

The toxicities of the crude solvent extracts obtained in the single solvent extractions are presented in Figure 1. From the results obtained is clear that all the crude solvent extracts present severe toxic effects to Vibrio fischeri with $\mathrm{EC}_{50}$ values varying from 0.0042 to $0.0114 \mathrm{mg}$ extract/L being the hexane crude extract the most toxic one. Hexane was the solvent that allowed to achieve the highest extraction yield in the pyrolysis chars and the higher amount of extract obtained can explain the highest toxicity. Also, the classes of compounds that were extracted with this solvent as well as some synergistic effects between them can be responsible for the higher toxicity.

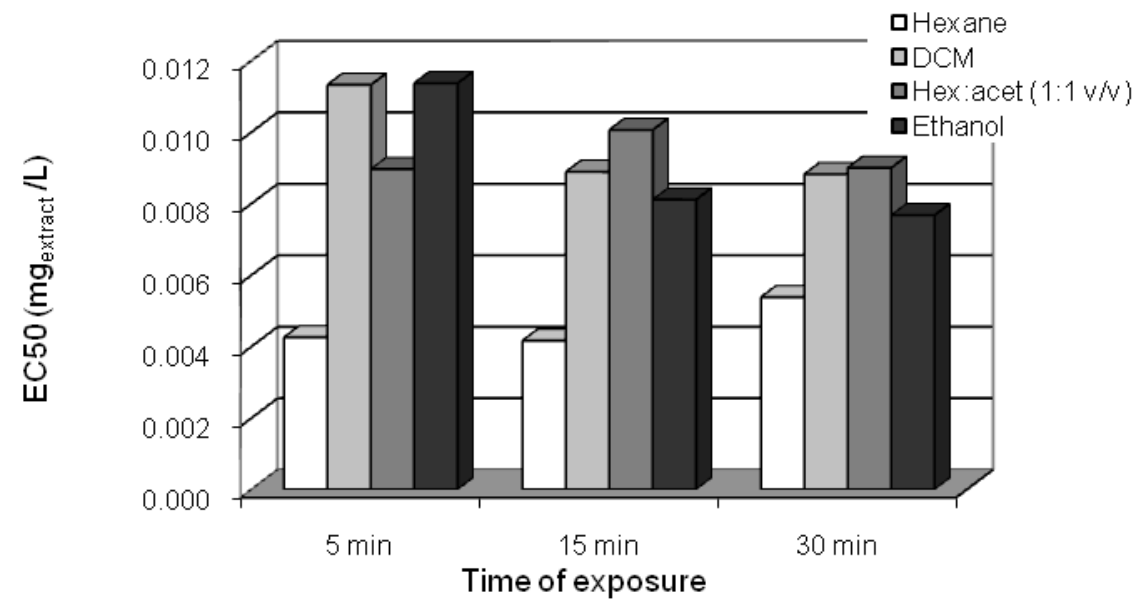

Figure 1. Toxicity data of crude solvent extract solutions. (Hexane - hexane crude extract; DCM - dichloromethane crude extract; Hex:Acet $(1: 1 \mathrm{v} / \mathrm{v})$ - crude extract obtained with the mixture 1:1 (v/v) of hexane and acetone; Ethanol - ethanol crude extract. 
The toxicities of each crude extract obtained in each of the extraction steps in the sequential extraction are presented in Table 4.

As expected, the toxicity decreases in each extraction step accordingly with the sucessive lower extraction yields obtained. The $1^{\text {st }}$ extraction step with hexane allowed to remove the most toxic contaminants and the subsequently extraction steps with the more polar solvents allowed to eliminate the residual polar contaminants that remained in the pyrolysis char that are less toxic to $V$. fischeri but neverthless, their contribution to the overall toxicity of the char is significant. This results suggest that could be useful to perform the sequential extraction for a better decontamination of the raw pyrolysis char.

Table 4. Toxicity data of crude extracts in the sequential extraction.

\begin{tabular}{cccc}
\hline \multirow{2}{*}{ Extraction step } & \multicolumn{3}{c}{ EC $_{50}(\mathrm{mg}$ extract/L) } \\
\cline { 2 - 4 } & $5 \mathrm{~min}$ & $15 \mathrm{~min}$ & $30 \mathrm{~min}$ \\
\hline 1 (Hexane) & 0.004 & 0.004 & 0.005 \\
2 (Hexane:acetone 1:1 v/v) & 0.031 & 0.044 & 0.045 \\
3 (Ethanol) & 0.147 & 0.131 & 0.150 \\
\hline
\end{tabular}

Table 5. List of aromatic compounds identified in the aromatic fraction of the hexane crude extract.

\begin{tabular}{ccc}
\hline Retention time (min) & Compound & $\begin{array}{c}\text { Relative concentration } \\
(\%)\end{array}$ \\
\hline 36.10 & Dimethylnaphthalene & 0.74 \\
38.76 & Diphenylmethane & 1.04 \\
40.16 & Trimethylnaphthalene isomer & 0.54 \\
40.45 & Trimethylnaphthalene isomer & 2.74 \\
41.02 & Trimethylnaphthalene isomer & 0.32 \\
41.24 & Trimethylnaphthalene isomer & 0.53 \\
43.79 & Isopropylmethylnaphthalene & 0.38 \\
45.12 & Diphenylpropane & 5.19 \\
45.84 & Isopropyldimethylnaphthalene & 1.09 \\
47.48 & Tetramethylnaphthalene & 2.01 \\
47.87 & Dimethylbiphenyl & 0.27 \\
48.31 & Isopropylbiphenyl & 1.17 \\
52.65 & Phenyldodecane & 1.68 \\
53.35 & Diphenylhexane & 1.00 \\
56.6 & Phenylnaphthalene & 0.54 \\
60.49 & Propenylanthracene & 1.00 \\
62.02 & Terphenyl & 2.45 \\
63.61 & Tetramethylphenanthrene & 2.22 \\
\hline
\end{tabular}

* Compound tentatively identified by comparison with the spectra of NIST and Wiley libraries.

\subsection{Fractionation of the hexane extract - Composition of the aliphatic and aromatic fractions}

The hexane crude extract was subsequently chosen to be chemically fractionated since it was the extract that presented the highest toxicity level and because it is interesting to study the chemical composition of the fractions in order to assess their contribution to the global toxicity.

In the GC chromatogram of the aliphatic fraction it can be seen a profile of $n$-alkanes from decane (C10) to triacontane (C30). Each $n$-alkane peak has coupled a smaller peak that corresponds to the homologous $n$-alkene. It can also be observed a group of lighter compounds, 
with retention times of 10 to 20 minutes, that corresponds mostly to branched and cyclic aliphatic hydrocarbons.

Concerning the aromatic fraction, the GC chromatogram showed several peaks that corresponds mostly to aromatic compounds (54.1\%) and aliphatic hydrocarbons (45.9\%). Thus, there were several aliphatics that were not eluted by hexane in the first fractionation step and only the second fractionation step with dichloromethane allowed to elute the remain aliphatic hydrocarbons.

In Table 5 is shown the aromatic compounds identified at sufficient concentration to enable the acquisition of elucidative mass spectra. Several other minor peaks that corresponds to substitute benzenes can be observed in the chromatogram. The aromatic compounds identified in this fraction were mainly, polyciclic aromatic hydrocarbons (PAHs) derivatives as well as phenyl derivatives. Among the PAHs, naphthalene derivatives were the predominants.

\section{CONCLUSIONS}

The results obtained in this study indicate that the more efficient extraction solvent to be used in the organic decontamination of chars obtained in the co-pyrolysis of plastics, tires and biomass waste is hexane. Higher extraction efficiency, concerning extraction yield and removing of toxic organic pollutants, was achieved with this solvent. A combination of extraction solvents, namely, an extraction with hexane followed by an extraction of hexane:acetone mixture, could be used to remove more efficiently different classes of organic contaminants.

The "petroleum like" profile of the aromatic fraction obtained in the fractionation procedure of the hexane crude extract can explain the high toxicity exhibited. It is plausible to assume that the compounds dominating quantitavely this fraction contribute to the observed toxicity.

Low molecular PAHs and in particular their alkylated or substituted congeners that are present in high concentrations in the aromatic fraction of the crude extract as well as the phenyl derivatives, present acute toxicity in the Microtox assay (Kaiser and Palabrica, 1991) so it could be argued that the highest toxicity level of the hexane extract can be attributed to the presence of these aromatic compounds more than to aliphatic hydrocarbons. However, it cannot be excluded that the compounds present in the aliphatic and polar fractions may contribute significantly to the toxicity of the crude extract. The aliphatic fraction was composed by a broad range of $n$-alkanes that by themselves cannot induce a marked toxic effect, but together with other compounds, some synergies may arise and originate a combined toxicity. The same can be applied to the polar fraction.

The char obtained after the organic decontamination still have significant amounts of some heavy metals, like $\mathrm{Pb}$ and $\mathrm{Zn}$ that can be leached which represents a problem concerning their safe reutilization and/or final disposal. In spite of previously demonstrated that the mobility of these heavy metals is suppressed in the extracted pyrolysis chars (Bernardo et al., 2010) the leachability of these heavy metals has to be monitored and strategies for removing them have to be evaluated.

\section{Acknowledgements}

The authors would like to acknowledge the Foundation for Science and Technology for supporting the Ph.D. grant of Maria Bernardo, which enabled this present work to be conducted. 


\section{REFERENCES}

Adachia K., Tainosho Y. (2004). Characterization of heavy metal particles embedded in tire dust. Environ. Int., 30, 1009- 1017.

APME (2010). PlasticsEurope - Association of Plastic Manufacturers in Europe. The Compelling Facts About Plastics 2009: An analysis of European plastics production, demand and recovery for 2008. Available at: http://www.plasticseurope.org; 2010.

Bernardo M., Lapa N., Gonçalves M., Barbosa R., Mendes B., Pinto F., Gulyurtlu I. (2010). Toxicity of char residues produced in the co-pyrolysis of different wastes. Waste Manage., 30, 628-635.

Buah W. K., Cunliffe A. M., Williams P. T. (2007). Characterization of products from the pyrolysis of municipal solid waste. Process Saf. Environ., 85, Issue 5, 450-457.

EC (2008). Directive 2008/98/EC of the European Parliament and of the council of 19 November 2008 on waste and repealing certain directives.

ETRMA (2010). European tyre and rubber manufacturers' association (ETRMA). End of life tyres - A valuable resource with growing potential - 2007 edition. Available at: http://www.etrma.org; 2010.

Helleur R., Popovic N., Ikura M., Stanciulescu M., Liu D. (2001). Characterization and potential applications of pyrolytic char from ablative pyrolysis of used tires. J. Anal. Appl. Pyrol., 5859, 813-824.

Hwang I. H., Ouchi Y., Matsuto T. (2007). Characteristics of leachate from pyrolysis residue of sewage sludge. Chemosphere, 68, 1913-1919.

Microtox (1992). Azur Environmental Microtox ${ }^{\circledR}$ system. Microtox ${ }^{\circledR}$ Omni - user manual. Microbics Corporation, Carlsbad, CA, USA, 1992.

Paradela F., Pinto F., Ramos A. M., Gulyurtlu I., Cabrita I. (2009). Study of the slow batch pyrolysis of mixtures of plastics, tyres and forestry biomass wastes. J. Anal. Appl. Pyrol., 85, 392-398.

USEPA (1996a). United States Environmental Protection Agency (USEPA). SW-846 Test Methods for Evaluating Solid Wastes. Physical/Chemical Methods. Method 3540C - Soxhlet Extraction. December 1996.

USEPA (1996b). United States Environmental Protection Agency (USEPA). SW-846 Test Methods for Evaluating Solid Wastes. Physical/Chemical Methods. Method 3611B - Alumina column cleanup and separation of petroleum wastes. December 1996.

Kaiser K. L.E. and Palabrica V. S. (1991). Photobacterium phosphoreum toxicity data index. Water Poll. Res. J. Canada, 26(3), 361-431

Kistler R. C., Widmer F., Brunner P. H. (1987). Behavior of chromium, nickel, copper, zinc, cadmium, mercury and lead during the pyrolysis of sewage sludge. Environ. Sci. Technol., 21, 704-708.

Zhang L., Xu C.C., Champagne P. (2010). Overview of recent advances in thermo-chemical conversion of biomass. Energ. Convers. Manage., 51, 969-982. 